\title{
Age-Related Regional Network of Magnetic Resonance Imaging Gray Matter in the Rhesus Macaque
}

\author{
Gene E. Alexander, ${ }^{1,9}$ Kewei Chen, ${ }^{2,9}$ Melaney Aschenbrenner, ${ }^{1,9}$ Tricia L. Merkley, ${ }^{1,9}$ Laura E. Santerre-Lemmon, ${ }^{1,9}$ \\ Jul Lea Shamy, ${ }^{3}$ William E. Skaggs, ${ }^{5,8}$ Michael H. Buonocore, ${ }^{4}$ Peter R. Rapp, ${ }^{3}$ and Carol A. Barnes ${ }^{5,6,7,8,9}$ \\ ${ }^{1}$ Department of Psychology, Arizona State University, Phoenix, Arizona 85287-1104, ${ }^{2}$ Positron Emission Tomography Center and Banner Alzheimer's \\ Institute, Banner Good Samaritan Medical Center, Phoenix, Arizona 85006, ${ }^{3}$ Fishberg Department of Neuroscience and Kastor Neurobiology of Aging \\ Laboratories, Mount Sinai School of Medicine, New York, New York 10029-6574, ${ }^{4}$ Department of Radiology, University of California, Davis, Sacramento, \\ California 95817, ${ }^{5}$ Arizona Research Laboratories, Division of Neural Systems, Memory and Aging, and Departments of ${ }^{6}$ Psychology and ${ }^{7}$ Neurology, and \\ Evelyn F. McKnight Brain Institute, University of Arizona, Tucson, Arizona 85724-5115, ${ }^{8}$ California National Primate Research Center, Davis, California \\ 95616, and ${ }^{9}$ Arizona Alzheimer's Consortium, Phoenix, Arizona 85006
}

Human structural neuroimaging studies have supported the preferential effects of healthy aging on frontal cortex, but reductions in other brain regions have also been observed. We investigated the regional network pattern of gray matter using magnetic resonance imaging (MRI) in young adult and old rhesus macaques (RMs) to evaluate age effects throughout the brain in a nonhuman primate model of healthy aging in which the full complement of Alzheimer's disease (AD) pathology does not occur. Volumetric T1 MRI scans were spatially normalized and segmented for gray matter using statistical parametric mapping (SPM2) voxel-based morphometry. Multivariate network analysis using the scaled subprofile model identified a linear combination of two gray matter patterns that distinguished the young from old RMs. The combined pattern included reductions in bilateral dorsolateral and ventrolateral prefrontal and orbitofrontal and superior temporal sulcal regions with areas of relative preservation in vicinities of the cerebellum, globus pallidus, visual cortex, and parietal cortex in old compared with young RMs. Higher expression of this age-related gray matter pattern was associated with poorer performance in working memory. In the RM model of healthy aging, the major regionally distributed effects of advanced age on the brain involve reductions in prefrontal regions and in the vicinity of the superior temporal sulcus. The age-related differences in gray matter reflect the effects of healthy aging that cannot be attributed to AD pathology, providing support for the targeted effects of aging on the integrity of frontal lobe regions and selective temporal lobe areas and their associated cognitive functions.

Key words: aging; voxel-based morphometry; multivariate analysis; monkey; prefrontal cortex; behavior

\section{Introduction}

Studies in human and nonhuman primates have suggested that frontal lobe-mediated cognitive processes, such as working memory, are preferentially affected by aging (West, 1996; Albert, 1997; Rapp and Gallagher, 1997; Rypma et al., 2001; Buckner, 2004; Petten et al., 2004; Grady et al., 2006). Human structural neuroimaging studies support the preferential effects of aging on frontal cortex, but have also shown effects in temporal, parietal, subcortical, and cerebellar regions (Raz et al., 1998; Good et al., 2001; Jernigan et al., 2001; Tisserand et al., 2002; Alexander et al., 2006). Such human studies, however, cannot exclude the possi-

\footnotetext{
Received April 24, 2007; revised Dec. 17, 2007; accepted Jan. 15, 2008.

This work was supported by National Institute on Aging Grants AG03376, AG025526, AG19610, and AG10606, California National Primate Research Center Base Grant RR-00016, the state of Arizona and Arizona Department of Health Services, and the Evelyn F. McKnight Brain Research Foundation. We thank the veterinary and technical staff of the California National Primate Research Center, as well as Michele Permenter, Julie Vogt, Matthew Archibeque, and Krista Hanson for technical assistance. A previous version of this manuscript was presented at the Society for Neuroscience meeting, San Diego, CA 2005

Correspondence should be addressed to Dr. Gene E. Alexander at his present address: Department of Psychology and the Evelyn F. McKnight Brain Institute, University of Arizona, Tucson, AZ 85721. E-mail: gene.alexander@arizona.edu.

DOI:10.1523/JNEUROSCI.1852-07.2008

Copyright $\odot 2008$ Society for Neuroscience $\quad$ 0270-6474/08/282710-09\$15.00/0
}

bility of underlying disease pathology for which clinical symptoms have not yet emerged. Brain regions, including parietotemporal and frontal association areas, that show prominent cerebral metabolic reductions in Alzheimer's disease (AD) with functional neuroimaging (Frackowiak et al., 1981; de Leon et al., 1983; Duara et al., 1986; Silverman et al., 2001; Alexander et al., 2002) also show decrements in individuals without clinical symptoms but with increased genetic risk for AD (Reiman et al., 1996, 2005).

Studying age-related brain changes in nonhuman primates provides a well established model of aging without the full complement of specific pathological features observed in $\mathrm{AD}$ (Kimura et al., 2003). Previous studies using structural magnetic resonance imaging (MRI) with rhesus macaques observed reductions in the caudate nucleus and putamen, but found no differences in hippocampus with increasing age (Matochik et al., 2000; Zhang et al., 2001; Shamy et al., 2006). These studies used manually traced volumes and univariate analyses for a limited set of brain structures. It is widely accepted that cognition depends on regional brain networks (Goldman-Rakic, 1988). Age-related alterations in brain networks may be especially important in determining the effects of aging on cognition (Burke and Barnes, 2006). 
Voxel-based morphometry (VBM) assesses structural differences throughout the brain with highly reliable methods (Ashburner et al., 2003). Multivariate network analyses, such as the scaled subprofile model (SSM) (Moeller et al., 1987), test for regional interactions that can reflect the distributed effects of aging or disease and associated alterations in functional or anatomical connectivity. The SSM has been applied in numerous human functional neuroimaging studies (Alexander and Moeller, 1994; Moeller and Eidelberg, 1997; Alexander et al., 1999; Habeck et al., 2003; Smith et al., 2006). Previous studies of healthy human aging using SSM analysis with structural MRI VBM identified age-related gray matter patterns that included reductions in dorsolateral prefrontal and temporal regions (Alexander et al., 2006; Brickman et al., 2007).

In the present study, we used SSM network analysis with MRI VBM in young adult and aged rhesus macaques. Two primary hypotheses were tested: (1) that brain regions known to be preferentially affected in healthy human aging, including prefrontal and temporal areas, would be reduced in older rhesus monkeys, as part of a regionally distributed network of age-related effects on gray matter volume; and (2) that individual differences in the expression of the age-related pattern would be associated with decrements in working memory performance, a cognitive ability known to be associated with age-related decline in humans (Grady and Craik, 2000).

\section{Materials and Methods}

Subjects. Nineteen rhesus macaques (Macaca mulatta) were included in the study, consisting of seven young (mean age $\pm \mathrm{SD}, 10.0 \pm 1.8$; range, 7.7-12.4 years; five males, two females) and 12 old (mean age \pm SD, $23.5 \pm 2.7$; range, $19.8-27.5$ years; eight males, four females) adults. The age groups differed in mean age $\left(t_{(17)}=11.64 ; p \leq 0.0001\right)$, but did not differ in distributions of gender (Fisher's exact test, $p=0.62$ ). The age of each monkey can be multiplied by a factor of 3 to provide an approximate comparison to human aging (Tigges et al., 1988). Most of the animals were housed in pairs and were provided environmental enrichment, including monitored socialization in a large pen, behavioral testing, and regular fruits and vegetables.

Standard rations of laboratory primate chow were provided on a twice-daily regimen without dietary restrictions. Water was provided in the home cage on an ad libitum basis and the primate colony was kept on an alternating $12 \mathrm{~h}$ light/dark cycle schedule. All experimental procedures were performed in accordance with National Institutes of Health guidelines and were approved by Institutional Animal Care and Use Committees at the California National Primate Research Center and the University of California, Davis.

Image acquisition. MRI scans were acquired with a 1.5 Tesla GE Signa Horizon LX NV/i scanner (GE Medical Systems, Waukesha, WI) at the University of California Davis Imaging Research Center (Sacramento, CA). Each animal was first anesthetized with ketamine $(20 \mathrm{mg} / \mathrm{kg}$, i.m. and atropine $(0.4 \mathrm{mg} / \mathrm{kg}, \mathrm{i} . \mathrm{m}$.). Head position was maintained throughout the scanning session using an MRI-compatible stereotaxic frame. Volumetric T1-weighted MRI scans were acquired in the coronal plane with 80 contiguous, 1 -mm-thick slices using a radiofrequency-spoiled gradient-recalled echo sequence [three-dimensional spoiled gradientrecalled acquisition in a steady state, repetition time, $21 \mathrm{~ms}$; echo time, $7.9 \mathrm{~ms}$ (full echo); flip angle, $30^{\circ}$; field of view, $16 \mathrm{~cm} \times 16 \mathrm{~cm}$; matrix, $256 \times 256$; number of excitations, 4 (with no phase wrap option), bandwidth, $15.63 \mathrm{kHz}$.

Image processing. The MRI scans were initially processed using MRIcro (Rorden and Brett, 2000) image-processing software to manually segment the total brain volume from the major surrounding nonbrain tissue. This "skull stripping" procedure was performed for each scan by a single individual (M.A.) with manual tracing of the total brain area on each coronal section combined over all slices to create a total brain mask for nonbrain tissue segmentation and to produce a measure of total brain volume (TBV). High inter-rater (0.98) and intra-rater (0.96) reliabilities for this total brain segmentation procedure were obtained using a subset of the young and old rhesus monkey scans. The scans were then processed using in-house developed software written with Matlab (MathWorks, Natick, MA) combined with the statistical parametric mapping (SPM2; Wellcome Department of Imaging Neuroscience, London, UK) program to implement optimized VBM preprocessing procedures (Ashburner and Friston, 2000; Good et al., 2001) and SSM network analysis for structural MRI on a voxel basis (Moeller et al., 1987; Alexander and Moeller, 1994; Alexander et al., 2006) adapted for the rhesus macaque.

Briefly, we first developed an iterative process to create a customized MRI template and its associated tissue priors using the 19 MRI scans to subsequently segment gray matter images after spatial normalization to the customized template. For the creation of the MRI template, we first selected a single representative scan from an initial subsample of four young and four old rhesus monkey MRI scans. The single scan served as a target image so that the segmented tissue maps for each of the eight MRI scans from the subsample could be spatially normalized to this single scan. The tissue segmentation for each of the eight scans started with a visual determination of the thresholds that distinguish the gray, white, and CSF components based on the original T1 images to provide initial tissue priors used to subsequently segment the images with the cluster analysis procedure based on the modified mixture model as implemented in SPM (Ashburner and Friston, 1997). The selection of tissue intensity values for use as a starting point with automated segmentation approaches has been used previously in MRI studies of the rhesus macaque (Andersen et al., 1999). Once segmented, each of the segmented tissue maps from the eight raw MRI scans were spatially normalized to the corresponding tissue maps from the single target MRI and the resulting normalization parameters were applied to each of the eight raw MRI scans.

The images from the eight spatially normalized scans were then averaged to provide tissue priors for subsequent customized template construction for the whole sample of 19 rhesus monkey MRI scans. In addition, the mean image from the eight spatially normalized images was smoothed with an $8 \mathrm{~mm}$ Gaussian filter to create an initial rhesus monkey spatial normalization template derived from representative young and old adult MRI scans. The template generation steps described above were then repeated to create a customized template for the full sample of 19 MRI scans using tissue segmentation priors derived from the eight scan subsample tissue priors, spatially normalizing each of the 19 scans to the eight scan subsample template, averaging the 19 spatially normalized images, and smoothing the resulting mean image to $8 \mathrm{~mm}$. Figure 1 shows the average whole-brain image from the full sample-specific customized MRI template for the rhesus monkey scans.

Using the full sample-specific template, each rhesus monkey MRI scan was processed following optimized VBM procedures, in which segmentation priors from the full sample template were used for spatial normalization and segmentation of the gray matter maps. Each gray matter map was then multiplied by the Jacobian determinant, modulating the voxel values to preserve volume information after spatial normalization. An 8 $\mathrm{mm}$ Gaussian kernel was then applied to the gray matter maps to provide voxel-based maps of smoothed gray matter volume. Quality assurance review was performed for the images produced at each step for template generation, image postprocessing, and analysis.

Behavioral testing procedures. The behavioral apparatus and testing procedures used in this study have been described in detail previously (Rapp and Amaral, 1991; O’Donnell et al., 1999; Rapp et al., 2003). Briefly, a modified Wisconsin General Test Apparatus (WGTA) (Harlow and Bromer, 1938) was used for all behavioral testing. The WGTA was composed of a chamber with vertical bars situated in front of a tray for stimulus presentation. The tray included three equally spaced wells, one of which was baited with a food reward during testing. Separate clear Plexiglas and opaque screens, controlled by the experimenter, were used to limit the animal's physical access to the wells and to impose retention interval delays during testing, respectively. A one-way mirrored screen allowed the tester to remain undetected while observing the animal's performance. Five young and 11 old monkeys received behavioral testing to assess aspects of recognition memory performance using delayed re- 
sponse (DR) and delayed nonmatching to sample (DNMS) tasks. The DR task assesses working memory performance over varying delay intervals, thought to primarily depend on frontal brain functions and rhesus monkeys have previously shown age-related reductions in this task (O'Donnell et al., 1999). The DNMS task assesses aspects of recognition memory for rule-based learning that has been associated with reduced performance because of lesions localized to the temporal lobe, as well as in relation to aging effects (Moss et al., 1988; Rapp and Amaral, 1989; Herndon et al., 1997; Buffalo et al., 2000; Saksida et al., 2006).

In the DR task, training began with a nodelay condition in which the subject observed the tester bait one of the two lateral wells of the stimulus tray with a food reward. Both wells were then covered and the subject was given the opportunity to respond after the clear Plexiglas screen was raised. The subject was allowed to retrieve the food reward after a correct response. The test sessions included 30 trials per day administered with a $20 \mathrm{~s}$ intertrial interval. During each test session, a pseudorandom order was used to assure that the left and right wells were baited with the food reward with equal frequency. After reaching the $90 \%$ correct criterion with the no-delay training (i.e., nine or fewer errors in nine consecutive 10-trial blocks), a $1 \mathrm{~s}$ delay was imposed by lowering the opaque screen of the WGTA between the baiting and response portion of each trial and the subjects were tested until they achieved the $90 \%$ correct criterion. The memory demands of the DR task were then increased by adding successively longer retention intervals of 5, 10, 15, 30,

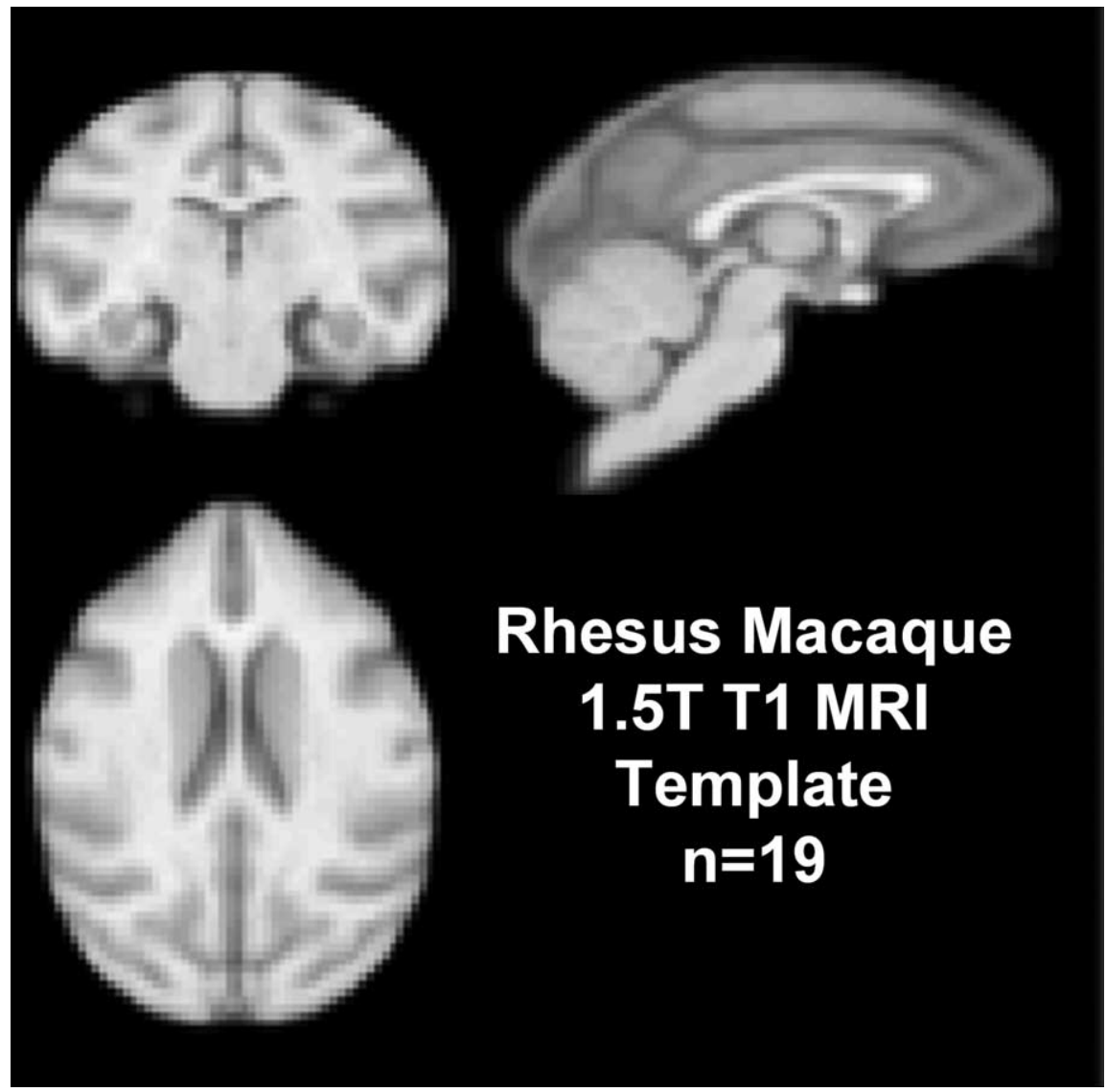

Figure 1. MRI template for the rhesus macaque. A customized MRI template was created for the sample of 19 MRI scans using SPM2 voxel-based morphometry and an iterative procedure using sample-specific segmentation priors adapted for the rhesus macaque. The average whole-brain template is shown in sagittal, coronal, and axial views.

and $60 \mathrm{~s}$. Testing for each retention interval in-

cluded a total of 90 trials with 30 trials administered per day.

In the DNMS task, each trial began with a sample object placed over the baited central well of the WGTA. After hiding the stimulus from view for a specified time interval, the sample object was presented together over the lateral wells with a novel stimulus that covered a food reward. Pairs of objects were drawn from a collection of 400 stimuli so that each trial consisted of unique stimulus pairs and rewarded items were presented over the left and right wells of the WGTA with equal frequency. Monkeys were tested with 20 trials per day for $5 \mathrm{~d}$ per week with a $10 \mathrm{~s}$ delay until performance reached a criterion of $90 \%$ correct over 100 trials. Once the nonmatching rule of the DNMS task was learned to criterion, testing proceeded with progressively longer retention intervals, including 15, 30, 60, and $120 \mathrm{~s}$ with 20 trials/d for $5 \mathrm{~d}$ for each interval, and $600 \mathrm{~s}$ with 5 trials/d for $10 \mathrm{~d}$.

Summary measures of task performance were computed as the average percentage correct across delay intervals for the DR (5-60 s) and DNMS (15-600 s) tasks (O’Donnell et al., 1999; Calhoun et al., 2004; Small et al., 2004; Shamy et al., 2006) to test associations with the age-related MRI SSM network pattern.

Statistical analysis of imaging and behavioral performance. SSM network analysis was subsequently performed on a voxel-basis for the VBM gray matter maps. The assumptions and procedures of the SSM have been described in detail previously (Moeller et al., 1987; Alexander and Moeller, 1994) and SSM has been previously applied to MRI VBM gray matter maps in human studies of aging (Alexander et al., 2006; Brickman et al., 2007). SSM analysis represents a modified form of principal component analysis (PCA) that identifies spatial covariance patterns in neuroimaging data related to external variables, such as demographic characteristics, cognitive performance, or biological and genetic factors.

In the SSM, mean voxel values across regions and subjects for the natural log transformed gray matter volume maps, at each voxel for each subject, are subtracted. These SSM transformations effectively normalize each voxel for total gray matter and subject differences before the PCA to extract the variance reflecting the region by subject interaction in the MRI data. We performed the SSM on VBM gray matter maps to identify patterns of gray matter associated with age in this sample of young and old rhesus macaques. Multiple-regression analyses were used to identify the best set of SSM component patterns predicting age group. Gender and TBV were subsequently added as initial covariates to the age group regression model to test for their potential influence on the SSM age group pattern. Subject scores for the observed age-related pattern were tested in relation to behavioral performance for the DR and DNMS tasks. For this purpose, a summary score for each procedure was calculated as the mean performance across all memory delays tested after initial task acquisition (O'Donnell et al., 1999; Calhoun et al., 2004; Shamy et al., 2006). These values were used to evaluate individual differences in pattern expression for the age-related network of MRI gray matter in the old rhesus monkeys and in both age groups combined. The behavioral specificity of correlates with age-related pattern expression was subsequently tested for both the DR and DNMS summary measures, after first controlling for task performance on DNMS and DR summary scores, respectively. A significance threshold of $p<0.05$ was used in the regression models to test the hypothesized associations between the age-related gray matter pattern and memory performance.

A bootstrap resampling procedure (Efron and Tibshirani, 1994) was performed with 500 iterations to provide reliability estimates for the observed SSM pattern weights associated with the age group difference in this sample. In the implementation of the bootstrap procedure for the SSM, the initial linearly combined pattern point estimate is divided by the SD from the bootstrap procedure for each voxel to produce a spatial covariance pattern with reliable estimates of the regional contributions to the network pattern of interest (Habeck et al., 2005). Regional minima 

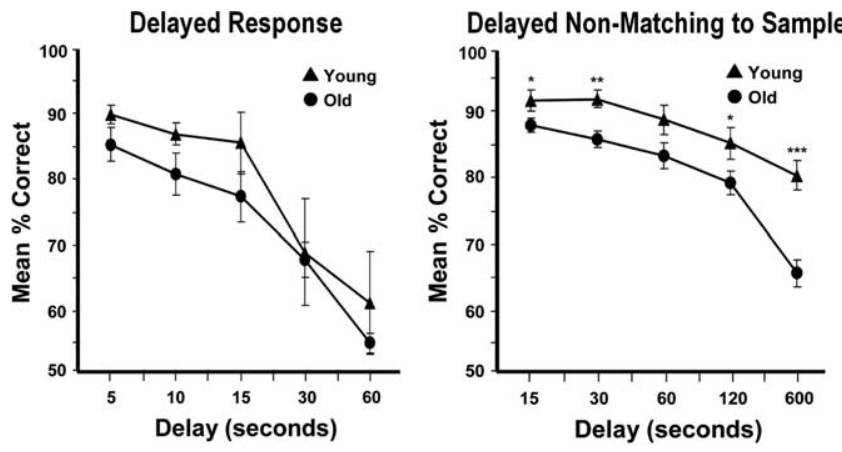

Figure 2. Behavioral performance comparing the young $(n=5)$ and elderly $(n=11)$ adult rhesus monkey groups on DR and DNMS tasks. Left, The comparison between age groups for the DR task with multiple retention intervals. Right, The age-group comparisons for the DNMS task for increasing retention intervals. The $y$-axes for both graphs show the percentage correct performance, whereas the retention intervals administered for each task are shown on the $x$-axes. Average performance with error bars indicating SEM is shown for each retention interval. Significant group differences using the nonparametric Mann-Whitney $U$ test are indicated by ${ }^{*} p \leq 0.05,{ }^{* *} p \leq 0.02$, and ${ }^{* * *} p \leq 0.002$.

and maxima voxels from the SSM bootstrapped image pattern weights were selected with a $Z$ estimate threshold value $\geq|2.5|$ and were superimposed on the full sample MRI T1 customized template for localization in relation to a standard neuroanatomical atlas of the rhesus monkey (Paxinos et al., 2000). The anatomical locations for the major contributing regions in the age-related network pattern were initially identified by a single individual (M.A.) and were subsequently confirmed by a second individual (G.A.) for each of the major pattern locations on the template.

Analyses of behavioral performance were performed using repeatedmeasures ANOVA and Huynh-Feldt adjustment with age group as the between-subject factor and retention interval as the within-subject factor. Follow up pairwise group comparisons for the individual retention intervals were performed with the nonparametric Mann-Whitney $(\mathrm{M}-\mathrm{W}) U$ test to address possible ceiling or floor effects when evaluating age group differences for the smaller subgroups receiving behavioral testing. Differences between the groups in age and gender for the total sample were tested by an unpaired $t$ test and Fisher's exact test, respectively.

\section{Results}

\section{Behavioral characterization of the sample}

The behavioral performances of the young $(n=5)$ and old $(n=$ 11 ) adult monkey groups are shown in Figure 2. For the DR task, there was a significant main effect for retention interval $\left(F_{(3,47)}=\right.$ $40.15, p \leq 0.0001)$ indicating a decline in performance with increasing retention interval in both groups. Despite an observable numerical difference, the group $\left(F_{(1,14)}=1.38, p=0.26\right)$ and group by retention interval interaction $\left(F_{(3,47)}=0.46, p=0.73\right)$ effects were not statistically significant (Fig. 2). The groups also did not differ in trials to criterion for the zero (mean \pm SD young trials, $115.8 \pm 86.2$; mean \pm SD old trials, $149.1 \pm 287.5 ; \mathrm{M}-\mathrm{W}$, $\mathrm{z}=-0.65, p=0.52$ ) and $1 \mathrm{~s}$ delays (mean \pm SD young trials, $70.0 \pm 121.2$; mean $\pm \mathrm{SD}$ old trials, $135.5 \pm 161.4 ; \mathrm{M}-\mathrm{W}, z=$ $-1.01, p=0.31$ ). Performance in the aged group was comparable with the impaired DR accuracy observed in larger numbers of aged subjects from the same study population (O'Donnell et al., 1999), consistent with findings from many other studies (Bartus et al., 1978; Arnsten and Contant, 1992; Arnsten and Jentsch, 1997), and among the relatively small groups available for the present analysis, the absence of a significant group effect was primarily attributable to variance in the test scores for the young adult group.

For the DNMS task, there were significant main effects of group $\left(F_{(1,14)}=16.81, p \leq 0.001\right)$ and retention interval $\left(F_{(3,47)}\right.$

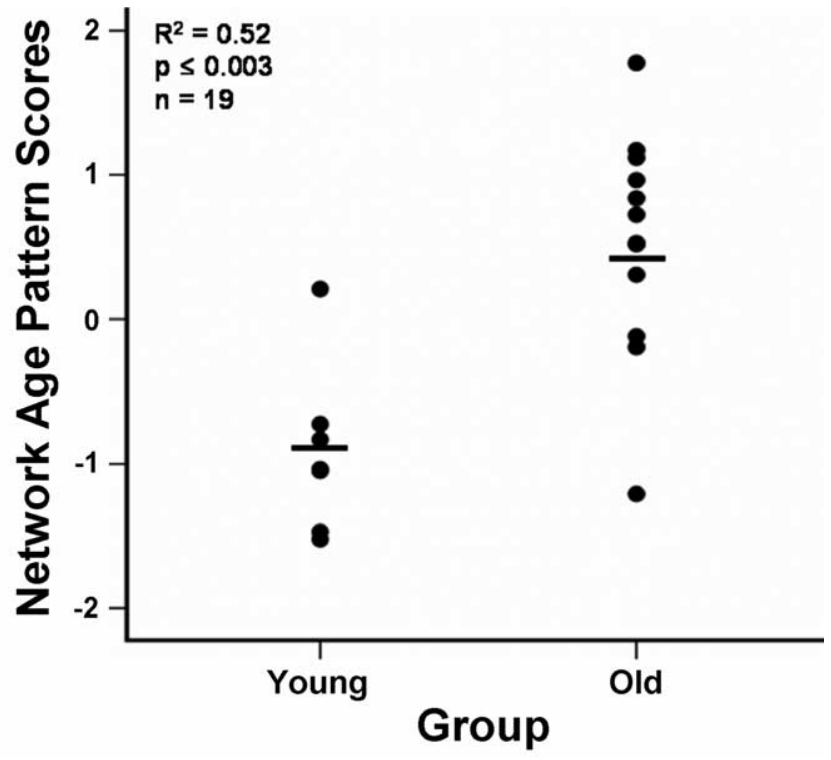

Figure 3. Multiple regression of SSM subject scores from the network analysis of MRI voxelbased morphometry in young adult $(n=7)$ and elderly $(n=12)$ rhesus macaques. The scatterplot shows that the aged monkey group has a higher expression of the MRI age-related network pattern than does the young monkey group. The age-related network subject scores were derived from the linear combination of the second and third SSM component patterns.

$=31.44, p \leq 0.0001)$, as well as a significant group by retention interval interaction $\left(F_{(3,47)}=3.10, p \leq 0.03\right)$, confirming the expected decline in performance with increasing retention that was greater in the old than young group. Follow-up group comparisons showed that old monkeys performed more poorly than the young subjects at the $15,30,120$, and 600 s retention intervals $(0.002 \leq p$ values $\leq 0.05)$ (Fig. 2$)$. Although the subjects scored at comparable criterion levels of accuracy before testing with increasingly challenging memory delays, the old monkeys (mean \pm SD trials, 895.5 \pm 361.9$)$ required more trials than did the young group (mean $\pm \mathrm{SD}$ trials, $221.8 \pm 144.8$ ) to reach criterion performance on the training condition with the $10 \mathrm{~s}$ retention inter$\operatorname{val}(\mathrm{M}-\mathrm{W}, z=-2.78, p \leq 0.006)$.

\section{Age-group differences in MRI gray matter}

The difference between the young and old rhesus monkey groups in the SSM analysis of the VBM processed MRI data were initially tested using a multiple regression model with SSM subject scores for the first eight component patterns, which accounted for the top $80 \%$ of the variance in the MRI data. This model, including all eight patterns, significantly predicted age group $\left(F_{(8,10)}=3.68\right.$, $p \leq 0.029$ ). A linear combination of the second and third component pattern subject scores was the best predictor of chronological age group, accounting for $52 \%$ of the variance $\left(F_{(2,16)}=\right.$ 8.62, $p \leq 0.003$ ) (Fig. 3). Subject scores for the two contributing component patterns each significantly predicted age group (pattern 2, $t_{(10)}=-2.44, p \leq 0.035$; pattern $3, t_{(10)}=-3.80, p \leq$ 0.003 ) with no other SSM patterns accounting for significant variance in the model. After entering $\operatorname{TBV}\left(F_{(1,17)}=1.01, p=\right.$ $0.33)$ and gender $\left(F_{(1,16)}=0.09, p=0.77\right)$ into the model, neither contributed significantly and the variance explained by the combination of the two SSM component patterns in age group was unchanged, accounting for $53.6 \%$ of the variance $\left(F_{(1,15)}=19.98\right.$, $p<0.0001)$. Bootstrap resampling of the linearly combined pattern of the second and third SSM components was characterized by gray matter volume reductions indicated by voxels with max- 


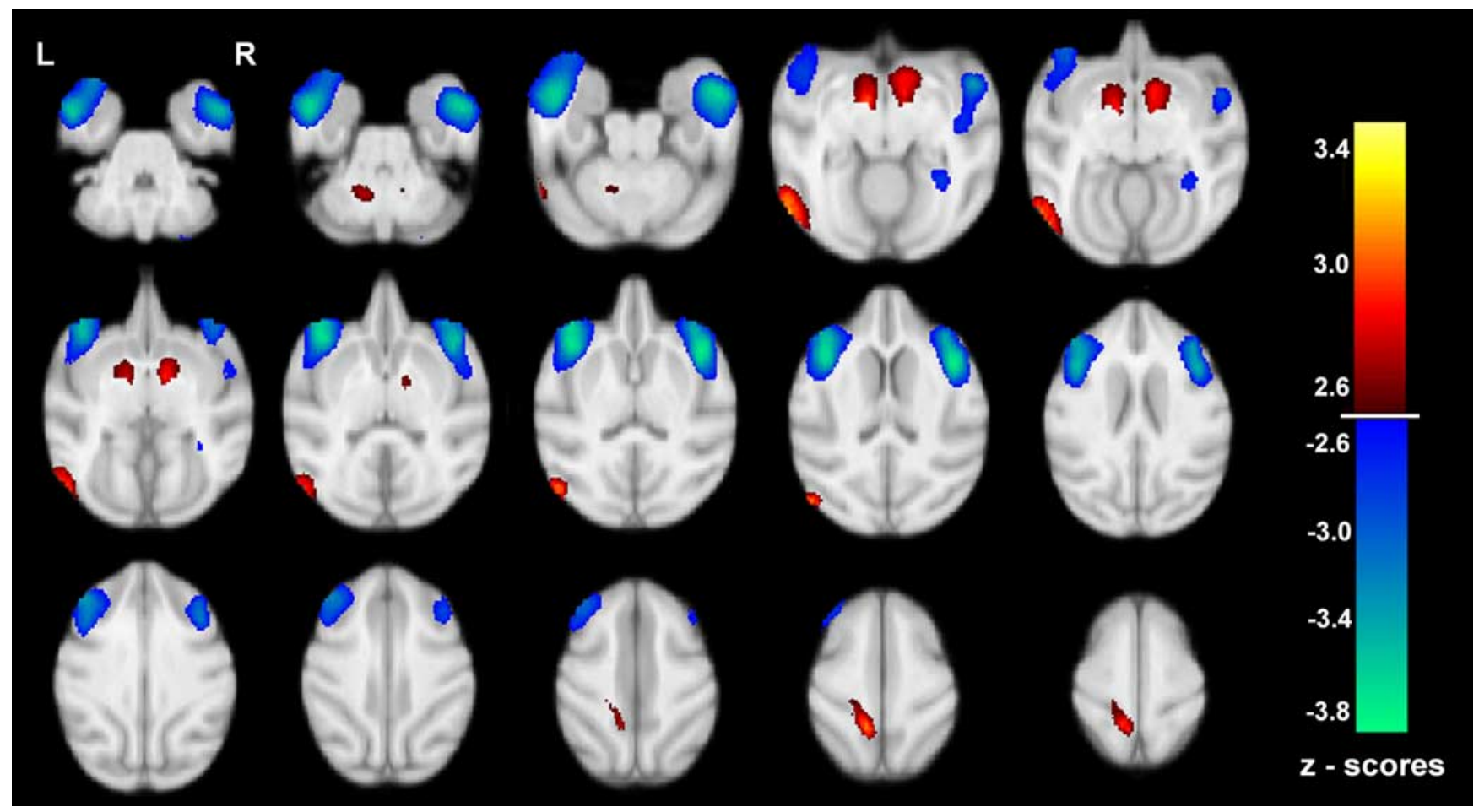

Figure 4. MRI gray matter pattern reflecting the linear combination of two SSM components whose subject scores predicted age group in the rhesus macaques. Voxels with SSM pattern weights are superimposed on axial slices from the SPM2 customized template for the rhesus macaque. The blue end of the color scale indicates brain regions showing lower gray matter volume with older age, whereas the orange end of the scale shows areas of relative increased gray matter with increasing age. Thus, a subject with a high positive score for this age-related pattern has relatively greater reductions in the blue areas and relatively greater covarying increases in the orange areas. Only voxels with z scores $\geq|2.5|$ after bootstrap resampling to provide robust regional pattern weights are shown. Notable regions of reduction (blue) with older age are observed (from top to bottom rows), mainly in the vicinities of the bilateral superior temporal sulcus, lateral fissure, and ventrolateral and dorsolateral prefrontal regions, whereas relative increases (orange) are seen in the vicinities of left cerebellum, left visual cortex, bilateral globus pallidus, and a left parietal region. That the older monkeys had a higher mean network age pattern score, with most of them having scores that were higher than the young and in the positive range, is consistent with a higher expression of the hypothesized age-related regional pattern in that group, including greater reductions in the blue areas than in the young. L, Left; R, right.

imal negative $z$ scores mainly occurring in the vicinities of bilateral dorsolateral prefrontal cortex $(-3.73 \leq z \leq-2.74)$, bilateral ventrolateral prefrontal and orbitofrontal sulcal areas $(-3.71 \leq$ $z \leq-2.73)$, a small right visual cortex region $(-2.84 \leq z \leq$ -2.72 ), and regions in the vicinities of bilateral superior temporal sulci $(-3.65 \leq z \leq-2.96)$ and lateral fissures $(-3.12 \leq z \leq$ -2.58 ). Areas with relative increases indicated by voxels with maximal positive $z$ scores were observed in vicinities of the bilateral globus pallidus $(2.73 \leq z \leq 2.83)$, a left visual cortex region $(2.94 \leq z \leq 3.32)$, an area in the left cerebellum $(z=2.74)$, and a region in the left parietal area $(z=2.97)$ (Fig. 4).

\section{Associations of age-related MRI gray matter network with behavioral performance}

In the total sample of rhesus macaques, poorer performance on the DR task summary score was associated with higher expression of the linearly combined SSM age-related pattern $(r=-0.64$, $\left.F_{(1,14)}=9.48, p \leq 0.008\right)$ (Fig. 5). The association between performance on the DNMS summary score and age-related pattern expression was not significant $\left(r=-0.41, F_{(1,14)}=2.87, p=\right.$ 0.11 ), but a higher number of trials to criterion during the training phase of the DNMS task was associated with greater expression of the age-related SSM pattern $\left(r=0.52, F_{(1,14)}=5.29, p \leq\right.$ 0.037). Trials to criterion during the zero and $1 \mathrm{~s}$ delay training conditions for the DR task were not related to age-related SSM pattern expression ( $p$ values $>0.55$ ).

After entering the DNMS summary measure into the regression model as an initial covariate, the association between the

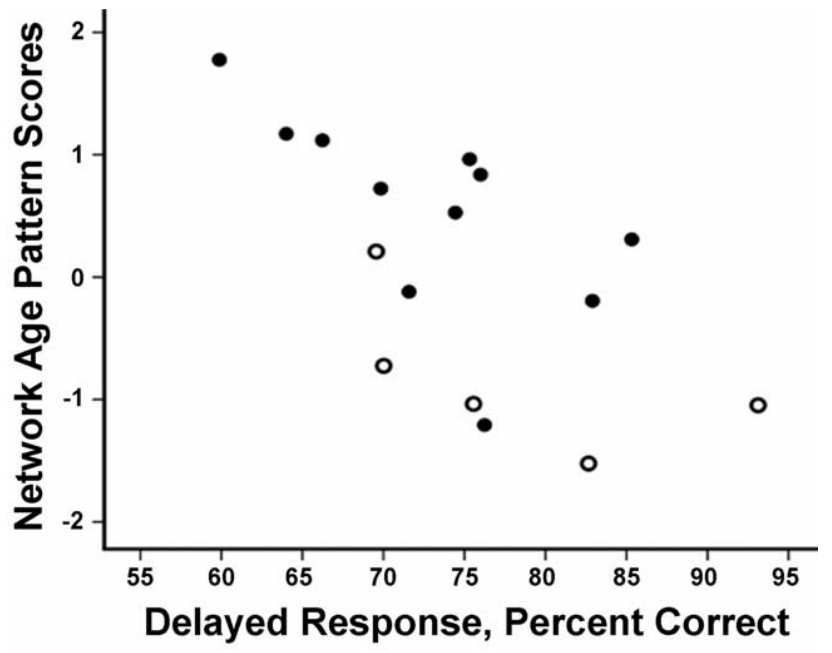

Figure 5. Regression analyses showing the association between behavioral performance on a DR task summary score with the subject scores from the age-related network SSM pattern. The old rhesus macaques are shown by filled circles and the young macaques have open circles in the scatterplot. A higher expression of the network age pattern is associated with poorer task performance for both the young and old groups combined $(r=-0.64, p \leq 0.008, n=16)$, as well as for the old macaque group alone $(r=-0.63, p \leq 0.038, n=11)$.

age-related pattern expression and performance on the DR task summary score remained significant $\left(r_{\text {partial }}=-0.59, F_{(1,13)}=\right.$ $6.88, p \leq 0.021)$. After we controlled for performance on the DR summary measure, the association between the age-related 
pattern expression and performance on the DNMS task summary score was not significant $\left(r_{\text {partial }}=-0.30, F_{(1,13)}=1.28, p=\right.$ $0.28)$.

In the old rhesus monkey group, higher expression of the linearly combined age-related pattern was associated with poorer performance on the DR summary score $\left(r=-0.63, F_{(1,9)}=5.93\right.$, $p \leq 0.038$ ) (Fig. 5). There was no association between the DNMS summary measure and the SSM age-related pattern subjects scores $\left(r=0.13, F_{(1,9)}=0.16, p=0.70\right)$. After we controlled for performance on the DNMS summary score, the association between the age-related pattern expression and performance on the DR summary score in the old group remained significant $\left(r_{\text {partial }}\right.$ $\left.=-0.64, F_{(1,8)}=5.41, p \leq 0.05\right)$. There was no significant association between DNMS and the SSM age-related pattern, after performance on the DR summary score was entered as an initial covariate $\left(r_{\text {partial }}=0.16, F_{(1,8)}=0.22, p=0.65\right)$. There were also no associations between trials to criterion during training on the DR or DNMS tasks with pattern expression in the old monkey group ( $p$ values $>0.56$ ).

\section{Discussion}

MRI assessed on a voxel basis in young and old adult rhesus macaques using multivariate SSM network analysis revealed a regionally distributed age-related pattern of gray matter volume showing reductions, including in dorsolateral prefrontal regions, ventrolateral prefrontal and orbitofrontal sulcal areas, and regions in the vicinities of the superior temporal sulcus and lateral fissure. This study represents the first application of network analysis with structural MRI to a nonhuman primate model of healthy aging to characterize the regional extent of aging effects throughout gray matter. The findings are consistent with previous studies of healthy aging in humans that have used region of interest and similar voxel-based univariate and multivariate methods, showing preferential effects of aging on selective prefrontal and temporal brain regions (Sullivan et al., 1995; Raz et al., 1998; Tisserand et al., 2002; Alexander et al., 2006; Brickman et al., 2007). Neuronal loss and disorganization in regions of the dorsolateral prefrontal cortex have been associated with agerelated cognitive effects in rhesus monkeys (Cruz et al., 2004; Smith et al., 2004). That we did not observe reductions in medial temporal lobe structures as part of the voxel-based age-related pattern is consistent with previous findings showing no agerelated reductions in manually traced hippocampal volumes in rhesus macaques (Shamy et al., 2006). These findings suggest that the reports of hippocampal reductions with similar MRI methods in healthy elderly humans may reflect very early effects of $\mathrm{AD}$ or increased risk for AD (Buckner, 2004).

With SSM network analysis, we found areas with relative increases in gray matter in vicinities of the cerebellum, globus pallidus, a small area in visual cortex, and a parietal region with increasing age. Previous studies of aging in the rhesus macaque reported reduced volumes in basal ganglia structures using manually defined regions, including in the caudate nucleus and putamen, but found no age-related reductions in globus pallidus (Zhang et al., 2001). In contrast, voxel-based network analysis did not identify reductions in the caudate or putamen as part of the age-related pattern. Previous studies reporting volume reductions in subregions of the basal ganglia have observed age effects when including adolescent monkeys (ages $<7$ years) in regression or group analyses for comparison with aged monkeys (ages $>20$ years), whereas studies including young to middle aged adults (ages 10-18 years) showed less observable differences relative to the aged group (Matochik et al., 2000, 2004; Zhang et al.,
2001). Although some reductions in caudate nucleus and putamen may continue to progress throughout the aging process, our findings comparing mature young adults with aged monkeys suggest that reductions in these basal ganglia subregions may not have a primary role in defining the major age-related regional decline that occurs in the transition from young adulthood to advanced aging in the rhesus monkey.

A previous study of healthy human aging using positron emission tomography (PET) with large regions of interest and the same PCA-based multivariate analysis used in the current study to measure regional cerebral glucose metabolism (rCMRglu) showed age-related patterns that included reductions in prefrontal brain regions with relatively higher metabolism in cerebellum, basal ganglia, and areas in occipital cortex (Moeller et al., 1996). Zuendorf et al. (2003) observed regions of hypometabolism using a voxel-based PCA analysis of PET rCMRglu in healthy human aging that included reductions in prefrontal cortex and caudate nucleus, but reported relative preservation in putamen, cerebellum, occipital cortex, thalamus, and primary motor cortex. In a nonhuman primate study of PET rCMRglu with selected frontal and temporal regions of interest, reductions in orbitofrontal glucose metabolism was associated with increasing age in a group of old adult rhesus macaques (Eberling et al., 1997).

Together with our previous and current MRI gray matter findings, these results suggest that reductions in frontal cortex function and structure with increasing age are most consistently observed in both human and nonhuman primates, whereas the nature of age-related alterations in basal ganglia subregions appear more variable. The relative increases observed in our study may reflect preservation of gray matter, but may also be related to age-related changes in brain shape or segmentation. Future longitudinal studies of the change in MRI gray matter morphology with age in the rhesus macaque may be helpful in clarifying the physiological implications of the observed aging effects in cortical and subcortical brain regions.

It is also noteworthy that findings from the current study showed bilateral age-related gray matter reductions in regions within the temporal poles, which were not observed in our previous MRI study in humans (Alexander et al., 2006). The temporal poles have been implicated in the recognition and processing of semantic knowledge in humans and socially meaningful vocalizations in nonhuman primates (Poremba et al., 2004; DehaeneLambertz et al., 2006; Zahn et al., 2007). Such cognitive processes remain preserved in healthy human aging. Our findings suggest the possibility that these and related abilities may be preferentially altered by aging in the rhesus macaque. Additional studies are needed to address this question.

We found prominent reductions in the dorsolateral prefrontal regions, ventrolateral prefrontal and orbitofrontal sulcal areas, and in vicinities of the superior temporal sulcus and lateral fissure with advanced aging in the rhesus monkey. It is possible that these frontal and temporal regions only begin to decline on MRI with the transition from mature young adulthood to advanced aging after alterations in other brain structures, like the caudate and putamen, occur in the transition from adolescence to young adulthood. Such a model of brain aging suggests a regionally distributed progression of preferentially affected brain regions that are diminished at differing rates during different stages of the aging process in the rhesus macaque. Alternatively, the prefrontal and superior temporal sulcal regions may decline monotonically throughout the course of aging in the rhesus monkey. Additional study is needed to evaluate how regional network patterns of gray matter differ when adolescent monkeys (ages $<7$ years) are in- 
cluded to identify the regional effects of aging over the full spectrum from very young to advanced aging.

The observed differences in age effects between studies may also be related to the methodological differences. Although VBM provides a highly reliable and unbiased approach for evaluating regional differences throughout the brain (Ashburner et al., 2003), tissue segmentation for voxel-based methods can be differentially sensitive to effects in some brain regions. It is possible that the lack of age-related reductions in the caudate and putamen, as well as the observed relative preservation in the vicinity of the globus pallidus may be related to differences in the quality of tissue segmentation for such subcortical structures. Studies using other tissue segmentation methods may further clarify this question. The associations between the SSM age-related network subject scores and DR task performance in our sample supports the hypothesis that the gray matter reductions in this study have behavioral consequences. Furthermore, the regional reductions in this study showed comparability with SSM VBM network analysis of age-related gray matter reductions in human studies, with regionally distributed patterns including reductions in dorsolateral prefrontal and perisylvian regions (Alexander et al., 2006, Brickman et al., 2007).

We found that individual differences in the expression of the MRI SSM pattern were associated with DR task performance, but not with DNMS summary scores. Both the DR and DNMS tasks involve memory functions that have been associated with age effects in rhesus monkeys (Rapp and Amaral, 1989; Rapp and Gallagher, 1997; Shamy et al., 2006). Whereas the DR task has been mainly associated with frontal lobe mediated working memory processing, the DNMS task involves aspects of recognition memory and has been shown to be specifically impaired with selective lesions in the perirhinal cortex (Buffalo et al., 2000; Saksida et al., 2006). Our findings suggest that individual differences in the expression of the age-related gray matter pattern are strongly related to differences in DR task performance. This finding was observed for both the total sample and when analyses were restricted to the aged monkey group, as well as after we statistically controlled for DNMS summary score performance. Although the age-related pattern was not significantly associated with the DNMS summary measure that averages performance across delays, more trials to reach criterion during training on this task, in which a $10 \mathrm{~s}$ delay is used, was associated with a higher expression of the age-related pattern in the total sample. This latter finding suggests that age-related reductions in the observed frontal and temporal brain regions may also have a role in diminishing the rate or efficiency of learning during acquisition of the DNMS task in which a shorter delay interval is used.

The aging process may lead to cognitive decline by altering vulnerable brain regions that affect the neural systems that support higher order cognitive abilities (Li et al., 2001; Tisserand and Jolles, 2003; Burke and Barnes, 2006). Healthy aging may exert its major effects on the brain and its associated cognitive functions through direct alterations of preferentially affected brain regions, brain areas which are not the primary site of aging effects but may be part of a distributed network that are altered through connections with directly affected vulnerable areas, or the efficiency of connections between such regions. It has been suggested that the aging brain may have a reserve capacity that must be sufficiently depleted for cognitive impairment to be observed (Stern et al., 1992, 1995, 2005; Alexander et al., 1997). Unlike the profound pathological effects of $\mathrm{AD}$, the comparatively subtle changes in cellular morphology, neurochemical function, microvascular plasticity, and synaptic transmission found in healthy aging may have major roles in the development of age-related cognitive decline (Barnes, 2001; Morrison and Hof, 2003; Burke and Barnes, 2006).

In addition, a growing number of human neuroimaging studies have suggested the potential importance of white matter changes in elucidating the effects of healthy aging on information processing (Good et al., 2001; Sullivan et al., 2001; Bartzokis et al., 2003; Sullivan and Pfefferbaum, 2006). Support for age-related alterations in frontal white matter integrity in rhesus macaques has been reported (Peters et al., 1996; Makris et al., 2007). Additional study is warranted to investigate the regional effects of aging on MRI white matter in the rhesus macaque and to test the potential association with the age-related gray matter pattern observed in this study.

That the full complement of the hallmark features of AD pathology is not observed in nonhuman primates provides the unique opportunity to test the effects of aging on the brain distinct from the potential contribution of incipient AD-type dementia. The similarity between the present findings and those showing prefrontal reductions in aging humans suggests a common age-related alteration in brain structure across primates. Furthermore, using the combination of MRI VBM with SSM network analysis in this nonhuman animal model of aging provides a method to evaluate regionally distributed age-related effects and to test individual differences caused by aging throughout the gray matter in relation to measures of behavior or other variables of interest. With SSM, the observed network pattern can be prospectively applied to independent samples and can be used to evaluate how pattern expression is altered by experimental manipulations in the same sample. Furthermore, the use of multivariate SSM network analysis provides a complement to univariate region of interest and voxel-based analyses that may further advance our understanding of the distributed neural systems affected by aging, help in tracking the progression of age effects, and may be potentially useful in evaluating interventions designed to delay or diminish the effects of brain aging and associated cognitive processes.

\section{References}

Albert MS (1997) The ageing brain: normal and abnormal memory. Philos Trans R Soc Lond B Biol Sci 352:1703-1709.

Alexander GE, Moeller JR (1994) Application of the Scaled Subprofile Model to functional imaging in neuropsychiatric disorders: a principal component approach to modeling regional patterns of brain function in disease. Hum Brain Mapp 2:79-94.

Alexander GE, Furey ML, Grady CL, Pietrini P, Brady DR, Mentis MJ, Schapiro MB (1997) Association of premorbid intellectual function with cerebral metabolism in Alzheimer's disease: implications for the cognitive reserve hypothesis. Am J Psychiatry 154:165-172.

Alexander GE, Mentis MJ, Van Horn JD, Grady CL, Berman KF, Furey ML, Pietrini P, Schapiro MB, Rapoport SI, Moeller JR (1999) Individual differences in PET activation of object perception and attention systems predict face matching accuracy. NeuroReport 10:1965-1971.

Alexander GE, Chen K, Pietrini P, Rapoport SI, Reiman EM (2002) Longitudinal PET evaluation of cerebral metabolic decline in dementia: a potential outcome measure in Alzheimer's disease treatment studies. Am J Psychiatry 159:738-755.

Alexander GE, Chen K, Merkley TL, Reiman EM, Caselli RJ, Aschenbrenner M, Lewis DJ, Pietrini P, Teipel SJ, Hampel H, Rapoport SI, Moeller JR (2006) Regional network of magnetic resonance imaging gray matter volume in healthy aging. NeuroReport 17:951-956.

Andersen AH, Zhang Z, Zhang M, Gash DM, Avison MJ (1999) Ageassociated changes in rhesus CNS composition identified by MRI. Brain Res 829:90-98.

Arnsten AF, Contant TA (1992) Alpha-2 adrenergic agonists decrease distractibility in aged monkeys performing the delayed response task. Psychopharmacology (Berl) 108:159-169. 
Arnsten AF, Jentsch JD (1997) The alpha-1 adrenergic agonist, cirazoline, impairs spatial working memory performance in aged monkeys. Pharmacol Biochem Behav 58:55-59.

Ashburner J, Friston KJ (1997) Multimodal image coregistration and partitioning: a unified framework. NeuroImage 6:209-217.

Ashburner J, Friston KJ (2000) Voxel-based morphometry: the methods. NeuroImage 11:805-821.

Ashburner J, Csernansky JG, Davatzikos C, Fox NC, Frisoni GB, Thompson PM (2003) Computer-assisted imaging to assess brain structure in healthy and diseased brains. Lancet Neurol 2:79-88.

Barnes CA (2001) Plasticity in the aging central nervous system. Int Rev Neurobiol 45:339-354.

Bartus RT, Fleming D, Johnson HR (1978) Aging in the rhesus monkey: debilitating effects on short-term memory. J Gerontol 33:858-871.

Bartzokis G, Cummings JL, Sultzer D, Henderson VW, Nuechterlein KH, Mintz J (2003) White matter structural integrity in healthy aging adults and patients with Alzheimer disease: a magnetic resonance imaging study. Arch Neurol 60:393-398.

Brickman AM, Habeck C, Zarahn E, Flynn J, Stern Y (2007) Structural MRI covariance patterns associated with normal aging and neuropsychological functioning. Neurobiol Aging 28:284-295.

Buckner RL (2004) Memory and executive function in aging and AD: multiple factors that cause decline and reserve factors that compensate. Neuron 44:195-208.

Buffalo EA, Ramus SJ, Squire LR, Zola SM (2000) Perception and recognition memory in monkeys following lesions of area TE and perirhinal cortex. Learn Mem 7:375-382.

Burke SN, Barnes CA (2006) Neural plasticity in the ageing brain. Nature Rev 7:30-40.

Calhoun ME, Mao Y, Roberts JA, Rapp PR (2004) Reduction in hippocampal cholinergic innervation is unrelated to recognition memory impairment in aged rhesus monkeys. J Comp Neurol 475:238-246.

Cruz L, Roe DL, Urbanc B, Cabral H, Stanley HE, Rosene DL (2004) Agerelated reduction in microcolumnar structure in area 46 of the rhesus monkey correlates with behavioral decline. Proc Natl Acad Sci USA 101:15846-15851.

Dehaene-Lambertz G, Dehaene S, Anton JL, Campagne A, Ciuciu P, Dehaene GP, Denghien I, Jobert A, Lebihan D, Sigman M, Pallier C, Poline JB (2006) Functional segregation of cortical language areas by sentence repetition. Hum Brain Mapp 27:360-371.

de Leon MJ, Ferris SH, George AE, Reisberg B, Christman DR, Kricheff II, Wolf AP (1983) Computed tomography and positron emission transaxial evaluations of normal aging and Alzheimer's disease. J Cereb Blood Flow Metab 3:391-394.

Duara R, Grady CL, Haxby JV, Sundaram M, Cutler NR, Heston L, Moore AM, Schlageter NL, Larson S, Rapoport SI (1986) Positron emission tomography in Alzheimer's disease. Neurology 36:879-887.

Eberling JL, Roberts JA, Rapp PR, Tuszynski MH, Jagust WJ (1997) Cerebral glucose metabolism and memory in aged rhesus macaques. Neurobiol Aging 18:437-443.

Efron B, Tibshirani RJ (1994) An introduction to the bootstrap. New York: CRC.

Frackowiak RSJ, Pozzilli C, Legg NJ, DuBoulay GH, Marshall J, Lenzi GL, Jones T (1981) Regional cerebral oxygen supply and utilization in dementia: a clinical and physiological study with oxygen-15 and positron emission tomography. Brain 104:753-778.

Goldman-Rakic PS (1988) Topography of cognition: parallel distributed networks in primate association cortex. Annu Rev Neurosci 11:137-156.

Good CD, Johnsrude IS, Ashburner J, Henson RNA, Friston KJ, Frackowiak RSJ (2001) A voxel-based morphometric study of ageing in 465 normal adult human brains. NeuroImage 14:21-36.

Grady CL, Craik FI (2000) Changes in memory processing with age. Curr Opin Neurobiol 10:224-231.

Grady CL, Springer MV, Hongwanishkul D, McIntosh AR, Winocur G (2006) Age-related changes in brain activity across the adult lifespan. J Cogn Neurosci 18:227-241.

Habeck C, Hilton HJ, Zarahn E, Flynn J, Moeller J, Stern Y (2003) Relation of cognitive reserve and task performance to expression of regional covariance networks in an event-related fMRI study of nonverbal memory. NeuroImage 20:1723-1733.

Habeck C, Krakauer JW, Ghez C, Sackeim HA, Eidelberg D, Stern Y, Moeller
JR (2005) A new approach to spatial covariance modeling of functional brain imaging data: ordinal trend analysis. Neural Comp 17:1602-1645.

Harlow HF, Bromer JA (1938) A test apparatus for monkeys. Psychol Rec 2:434-436.

Herndon JG, Moss MB, Rosene DL, Killiany RJ (1997) Patterns of cognitive decline in aged rhesus monkeys. Behav Brain Res 87:25-34.

Jernigan TL, Archibald SL, Fennema-Notestine C, Gamst AC, Stout JC, Bonner J, Hesselink JR (2001) Effects of age on tissues and regions of the cerebrum and cerebellum. Neurobiol Aging 22:581-594.

Kimura N, Tanemura K, Nakamura S, Takashima A, Ono F, Sakakibara I, Ishii Y, Kyuwa S, Yoshikawa Y (2003) Age-related changes of Alzheimer's disease-associated proteins in cynomolgus monkey brains. Biochem Biophys Res Commun 310:303-311.

Li SC, Lindenberger U, Sikstrom S (2001) Aging cognition: from neuromodulation to representation. Trends Cogn Sci 5:479-486.

Makris N, Papadimitriou GM, van der Kouwe A, Kennedy DN, Hodge SM, Dale AM, Benner T, Wald LL, Wu O, Tuch DS, Caviness VS, Moore TL, Killiany RJ, Moss MB, Rosene DL (2007) Frontal connections and cognitive changes in normal aging rhesus monkeys: a DTI study. Neurobiol Aging 28:1556-1567.

Matochik JA, Chefer SI, Lane MA, Woolf RI, Morris ED, Ingram DK, Roth GS, London ED (2000) Age-related decline in striatal volume in monkeys as measured by magnetic resonance imaging. Neurobiol Aging 21:591-598.

Matochik JA, Chefer SI, Lane MA, Roth GS, Mattison JA, London ED, Ingram DK (2004) Age-related decline in striatal volume in rhesus monkeys: assessment of long-term calorie restriction. Neurobiol Aging 25:193-200.

Moeller JR, Eidelberg D (1997) Divergent expression of regional metabolic topographies in Parkinson's disease and normal ageing. Brain 120:2197-2206.

Moeller JR, Strother SC, Sidtis JJ, Rottenberg DA (1987) Scaled subprofile model: a statistical approach to the analysis of functional patterns in positron emission tomographic data. J Cereb Blood Flow Metab 7:649-658.

Moeller JR, Ishikawa T, Dhawan V, Spetsieris P, Mandel F, Alexander GE, C Grady, Pietrini P, Eidelberg D (1996) The metabolic topography of normal aging. J Cereb Blood Flow Metab 16:385-398.

Morrison JH, Hof PR (2003) Changes in cortical circuits during aging. Clin Neurosci 2:294-304.

Moss MB, Rosene DL, Peters A (1988) Effects of aging on visual recognition memory in the rhesus monkey. Neurobiol Aging 9:495-502.

O’Donnell KA, Rapp PR, Hof PR (1999) Preservation of prefrontal cortical volume in behaviorally characterized aged macaque monkeys. Exp Neurol 160:300-310.

Paxinos G, Huang X-F, Toga AW (2000) The rhesus monkey brain in stereotaxic coordinates. San Diego: Academic.

Peters A, Rosene DL, Moss MB, Kemper TL, Abraham CR, Tigges J, Albert MS (1996) Neurobiological bases of age-related cognitive decline in the rhesus monkey. J Neuropathol Exp Neurol 55:861-874.

Petten CV, Plante E, Davidson PSR, Kuo TY, Bajuscak L, Glisky EL (2004) Memory and executive function in older adults: relationships with temporal and prefrontal gray matter volumes and white matter hyperintensities. Neuropsychologia 42:1313-1335.

Poremba A, Malloy M, Saunders RC, Carson RE, Herscovitch P, Mishkin M (2004) Species-specific calls evoke asymmetric activity in the monkey's temporal poles. Nature 427:448-451.

Rapp PR, Amaral DG (1989) Evidence for task-dependent memory dysfunction in the aged monkey. J Neurosci 9:3568-3576.

Rapp PR, Amaral DG (1991) Recognition memory deficits in a subpopulation of aged monkeys resemble the effects of medial temporal lobe damage. Neurobiol Aging 12:481-486.

Rapp PR, Gallagher M (1997) Toward a cognitive neuroscience of normal aging. Adv Cell Aging Gerontol 2:1-21.

Rapp PR, Morrison JH, Roberts JA (2003) Cyclic estrogen replacement improves cognitive function in aged ovariectomized rhesus monkeys. J Neurosci 23:5708-5714.

Raz N, Gunning-Dixon FM, Head D, Dupuis JH, Acker JD (1998) Neuroanatomical correlates of cognitive aging: evidence from structural magnetic resonance imaging. Neuropsychologia 12:95-114.

Reiman EM, Caselli RJ, Yun LS, Chen K, Bandy D, Minoshima S, Thibodeau SN, Osborne D (1996) Preclinical evidence of Alzheimer's disease in 
persons homozygous for the epsilon 4 allele for apolipoprotein E. N Engl J Med 334:752-758.

Reiman EM, Chen K, Alexander GE, Caselli RJ, Dandy D, Osborne D, Saunders AM, Hardy J (2005) Correlations between apolipoprotein E e4 gene dose and lower brain-imaging measurements of regional glucose metabolism. Proc Natl Acad Sci USA 102:8299-8302.

Rorden C, Brett M (2000) Stereotaxic display of brain lesions. Behav Neurol 12:191-200.

Rypma B, Prabhakaran V, Desmond JE, Gabrieli JDE (2001) Age differences in prefrontal cortical activity in working memory. Psychol Aging 16:371-384.

Saksida LM, Bussey TJ, Buckmaster CA, Murray EA (2006) No effect of hippocampal lesions on perirhinal cortex-dependent feature-ambiguous visual discriminations. Hippocampus 16:421-430.

Shamy JL, Buonocore MH, Makaron LM, Amaral DG, Barnes CA, Rapp PR (2006) Hippocampal volume is preserved and fails to predict recognition memory impairment in aged rhesus monkeys (Macaca mulatta). Neurobiol Aging 27:1405-1415.

Silverman DH, Small GW, Chang CY, Lu CS, Kung de Aburto MA, Chen W, Czernin J, Rapoport SI, Pietrini P, Alexander GE, Schapiro MB, Jagust WJ, Hoffman JM, Welsh-Bohmer KA, Alavi A, Clark CM, Salmon E, de Leon MJ, Mielke R, Cummings JL, et al. (2001) Neuroimaging in evaluation of dementia: regional brain metabolism and long-term outcome. JAMA 286:2120-2127.

Small SA, Chawla MK, Buonocore M, Rapp PR, Barnes CA (2004) Imaging correlates of brain function in monkeys and rats isolates a hippocampal subregion differentially vulnerable to aging. Proc Natl Acad Sci USA 101:7181-7186.

Smith DE, Rapp PR, McKay HM, Roberts JA, Tuszynski MH (2004) Memory impairment in aged primates is associated with focal death of cortical neurons and atrophy of subcortical neurons. J Neurosci 24:4373-4381.

Smith JF, Chen K, Johnson SC, Morrone-Strupinsky J, Reiman EM, Nelson A, Moeller JR, Alexander GE (2006) Network analysis of single-subject fMRI during a finger opposition task. NeuroImage 32:325-332.

Stern Y, Alexander GE, Prohovnik I, Mayeux R (1992) Inverse relationship between education and parietotemporal perfusion deficit in Alzheimer's disease. Ann Neurol 32:371-375.
Stern Y, Alexander GE, Prohovnik I, Stricks L, Link B, Lennon MC, Mayeux R (1995) Relationship between lifetime occupation and parietal flow: implications for a reserve against Alzheimer's disease pathology. Neurology 45:55-60.

Stern Y, Habeck C, Moeller J, Scarmeas N, Anderson KE, Hilton HJ, Flynn J, Sackeim H, van Heertum R (2005) Brain networks associated with cognitive reserve in healthy young and old adults. Cereb Cortex 15:394-402.

Sullivan EV, Pfefferbaum A (2006) Diffusion tensor imaging and aging. Neurosci Biobehav Rev 30:749-761.

Sullivan EV, Marsh L, Mathalon DH, Lim KO, Pfefferbaum A (1995) Agerelated decline in MRI volumes of temporal lobe gray matter but not hippocampus. Neurobiol Aging 16:591-606.

Sullivan EV, Adalsteinsson E, Hedehus M, Ju C, Moseley M, Lim KO, Pfefferbaum A (2001) Equivalent disruption of regional white matter microstructure in ageing healthy men and women. NeuroReport 12:99-104.

Tigges J, Gordon TP, McClure HM, Hall EC, Peters A (1988) Survival rate and life span of rhesus monkeys at the Yerkes regional primate research center. Am J Primatol 15:263-273.

Tisserand DJ, Jolles J (2003) On the involvement of prefrontal networks in cognitive ageing. Cortex 39:1107-1128.

Tisserand DJ, Pruessner JC, Sanz Argita EJ, van Boxtel MP, Evans AC, Jolles J, Uylings HBM (2002) Regional frontal cortical volumes decrease differentially in aging: an MRI study to compare volumetric approaches and voxel-based morphometry. Neuroimage 17:657-669.

West RL (1996) An application of prefrontal cortex function theory to cognitive aging. Psychol Bull 120:272-292.

Zahn R, Moll J, Krueger F, Huey ED, Garrido G, Grafman J (2007) Social concepts are represented in the superior anterior temporal cortex. Proc Natl Acad Sci USA 104:6430-6435.

Zhang Z, Andersen A, Grondin R, Barber T, Avison R, Gerhardt G, Gash D (2001) Pharmacological MRI mapping of age-associated changes in basal ganglia circuitry of awake rhesus monkeys. Neuroimage 14:1159-1167.

Zuendorf G, Kerrouche N, Herholz K, Baron J-C (2003) Efficient principal component analysis for multivariate $3 \mathrm{D}$ voxel-based mapping of brain functional imaging data sets as applied to FDG-PET and normal aging. Hum Brain Mapp 18:13-21. 